\title{
The clinical usefulness of non-invasive prenatal testing in pregnancies with abnormal ultrasound findings
}

\author{
Hyeyeon Boo', So Yun Kim², Eui Sun Seoung², Min Hyung Kim², Moon Young Kim², Hyun Mee Ryu², You Jung Han², and \\ Jin Hoon Chung ${ }^{2, *}$ \\ ${ }^{1}$ Department of Obstetrics and Gynecology, Hanyang University Guri Hospital, Hanyang University College of Medicine, Guri, Korea \\ ${ }^{2}$ Department of Obstetrics and Gynecology, Cheil General Hospital and Women's Healthcare Center, Dankook University College of Medicine, \\ Seoul, Korea
}

Purpose: This study aimed to evaluate the clinical usefulness of non-invasive prenatal testing (NIPT) as an alternative testing of invasive diagnostic testing in pregnancies with ultrasound abnormalities.

Materials and Methods: This was a retrospective study of pregnant women with abnormal ultrasound findings before 24 weeks of gestation between April 2016 and March 2017. Abnormal ultrasound findings included isolated increased nuchal translucency, structural anomalies, and soft markers. The NIPT or diagnostic test was conducted and NIPT detected trisomy 21 (T21), T18, T13 and sex chromosomal abnormalities. We analyzed the false positive and residual risks of NIPT based on the ultrasound findings.

Results: During the study period, 824 pregnant women had abnormal ultrasound findings. Among the study population, 139 patients $(16.9 \%)$ underwent NIPT. When NIPT was solely performed in the patients with abnormal ultrasound findings, overall false positive risk was $2.2 \%$ and this study found residual risks of NIPT. However, the discordant results of NIPT differed according to the type of abnormal ultrasound findings. Discordant results were significant in the group with structural anomalies with $4.4 \%$ false positive rate. However, no discordant results were found in the group with single soft markers.

Conclusion: This study found different efficacy of NIPT according to the ultrasound findings. The results emphasize the importance of individualized counseling for prenatal screening or diagnostic test based on the type of abnormal ultrasound.

Key words: Non-invasive prenatal test, Cell-free nucleic acids, Prenatal diagnosis, Ultrasonography, Congenital abnormalities.

\section{Introduction}

Non-invasive prenatal testing (NIPT) for aneuploidy screening has been available in the clinical field since 2011. The introduction of NIPT rapidly altered prenatal screening regimens [1-4] and significantly reduced the uptake of other screening and diagnostic tests [5-8]. Tests are usually applied to detect common chromosome aneuploidy such as trisomy 21 (T21), T18, and T13 with high sensitivity and specificity [1]. Currently, the American College of Obstetricians and Gynecologists (ACOG) and the Society for Maternal-Fetal Medicine (SMFM) both recommend to offer the option for aneuploidy screening including NIPT or diagnostic testing for fetal genetic disorders to every woman $[9,10]$.

Received: 21 November 2018, Revised: 18 December 2018, Accepted: 19 December 2018, Published: 31 December 2018

${ }^{*}$ Corresponding author: Jin Hoon Chung, M.D., Ph.D. (iD http://orcid.org/0000-0002-5137-0680

Department of Obstetrics and Gynecology, Cheil General Hospital and Women's Healthcare Center, Dankook University College of Medicine, 17 Seoae-ro 1-gil, Jung-gu, Seoul 04619, Korea.

Tel: +82-2-2000-7273, Fax: +82-2-2278-4574, E-mail:sabi0515@hanmail.net

Conflict of interest: The authors declare that they do not have any conflicts of interest.

(c) This is an open-access article distributed under the terms of the Creative Commons Attribution Non-Commercial License (http://creativecommons.org/licenses/by-nc/4.0/) which permits unrestricted non-commercial use, distribution, and reproduction in any medium, provided the original work is properly cited. 
On account of increased uptake and advanced screening performance of NIPT, the role of prenatal ultrasound has been reconsidered. However, the ACOG committee opinion still states to consider baseline ultrasound examination with NIPT to detect other structural anomalies [11]. Since the presence of fetal structural anomalies increase the risk of fetal aneuploidy [9], the findings can change the counseling method and the patient's choice between the screening or invasive diagnostic test $[3,11]$. Furthermore, the chromosomal abnormalities associated with fetal anomalies contain aberrations not routinely detected with NIPT. Therefore, the residual risk for a chromosome abnormality after negative NIPT results is higher when structural anomaly exists [10,12-15]. However, some women with increased risk of chromosomal abnormalities due to abnormal ultrasound findings might still consider NIPT as an alternative method for invasive diagnostic testing. Counseling technique with accurate information is more important for these women.

Although previous studies found the residual risk of NIPT in pregnancy with abnormal ultrasound findings, not enough studies have analyzed the discordant results and clinical utility of NIPT according to the type of abnormality in ultrasound. Thus, this study aimed to analyze the usefulness and actual efficiency of NIPT in the clinical field, according to the type of ultrasound findings in Korean population.

\section{Materials and Methods}

This study retrospectively reviewed medical record of all pregnant women with abnormal ultrasound findings in Cheil General Hospital and Women's Healthcare Center between April 2016 and March 2017. This study was reviewed and exempted from approval by the institutional review board at Cheil General Hospital and Women's Healthcare Center, Seoul, Republic of Korea (IRB no. CGH-IRB-2017-52) because the study only involved the existing data and the medical information was recorded in the way that participants could not be identified.

We reviewed the prenatal ultrasound database for ultrasound examinations performed before 24 weeks of gestation during this period. Women with singleton and twin pregnancies who underwent routine ultrasound examination with any of abnormal ultrasound findings were included in the study. The study population had screening or diagnostic test after detecting the ultrasound abnormalities. We analyzed demographic characteristics, pregnancy outcomes, choice and result of prenatal screening or diagnostic test, and prenatal ultrasound results with medical records.
Every patient in this study received pretest counseling after finding abnormality in ultrasound. We offered the choices among the testing options afterward, which included screening with maternal serum markers, screening with NIPT, invasive diagnostic test, and no screening test at all. A member or staff of the Obstetrics department performed the pretest counseling, which included detailed description of each test such as characteristics, methods, benefits, disadvantages, expected test performance, and potential adverse effects. The procedurerelated risk of miscarriage after invasive diagnostic test was also informed. The patients and partners made the decision after the counseling. We provided written information about the test when patient decided to have NIPT or invasive diagnostic test as a first-tier test. Since this study focused on the clinical utility of NIPT, patients who chose to have no further test other than the screening with maternal serum markers were not analyzed.

The physicians from departments of Radiology and Obstetrics performed routine ultrasound examinations. Ultrasound examination was performed in the first trimester between 11 weeks and 13 weeks 6 days of gestation. The first trimester ultrasound screening included nuchal translucency measurement and systemic anatomical evaluation of the fetus following the guidelines of the International Society of Ultrasound in Obstetrics and Gynecology (ISUOG) [16]. Second trimester detailed ultrasound examination between 20 weeks and 24 weeks of gestation evaluated further fetal systemic anatomy. Abnormal ultrasound findings in each examination included the increased nuchal translucency (INT), isolated or multiple structural anomalies, and soft markers. The INT included nuchal translucency measuring larger than 95th percentile for given gestational age [17]. Soft markers in this study included echogenic intracardiac foci, pyelectasis, echogenic bowel, thickened nuchal fold, ventriculomegaly, choroid plexus cyst, and short femur or humerus length [18]. Any isolated or multiple structural abnormalities commonly described in the literature were described as structural anomalies [19].

NIPT in this study detected risks for T21, T18, T13, and sex chromosomal abnormalities (SCA). Invasive diagnostic tests confirmed the abnormal results of NIPT. The result of NIPT was described as high risk, low risk, and no call result. Newborns physical examination and any genetic testing performed confirmed the chromosomally normal results. Newborn with a normal physical examination was considered as euploid. Invasive diagnostic tests included chorionic villus sampling, amniocentesis, or cordocentesis. Fetal genotyping was performed using the microscopic G-band karyotyping and the quantitative fluores- 
cent polymerase chain reaction. Previously descripted clinically significant chromosomal aberrations that are not detectable with NIPT [14], such as microdeletion/duplication, translocation, and mosaicism, were considered as a residual risk of NIPT and the frequency was calculated in this study.

In data analysis, categorical variables are shown as frequency (percentage) and were compared using the chi-square test or Fisher's exact test. Continuous variables are shown as mean and standard deviation and groups were compared using the Student's $t$-test. Statistical significance was concluded at a $P$ value $<0.05$.

\section{Results}

During the study period, 824 pregnant women had abnormal ultrasound findings. Among the study population, 139 (16.9\%) patients underwent NIPT as a first-tier test and 206 (25.0\%) patients had invasive diagnostic test. Since this study focused on the clinical usefulness of NIPT as an alternative to the invasive diagnostic test, the other 358 (43.4\%) patients who chose not to have further tests beside the screening with maternal serum markers and 121 (14.7\%) patients who were lost to follow-up for the further screening or diagnostic tests were excluded in further analysis. As a result, we investigated 345 patients who had NIPT or invasive diagnostic test. The abnormal ultrasound findings in study population included 146 (42.3\%) cases of INT, $70(20.3 \%)$ cases of structural anomalies, and 129 (37.4\%) of soft markers. Structural anomalies included 62 (88.6\%) isolated anomalies and 8 (11.4\%) multiple anomalies. Among the patients with soft markers, 117 (90.7\%) cases had only a single soft marker and 12 (9.3\%) cases had more than two soft markers. The Table 1 summarizes demographics and abnormal ultrasound findings of each group according to the first-tier choice of genetic test.

The NIPT results were low risk in 132 (95.0\%) cases and high risk in 7 (5.0\%) cases. Chromosomal aneuploidies suspected by NIPT were 4 cases of T21, one case of T18, and 2 cases of SCA. Diagnostic test was done in all 7 cases. Among patient with high risk NIPT results, 3 cases of T21 and one case of T18 had concordant results in the invasive diagnostic test. However, the other high-risk cases, one case of T21 and 2 cases of SCA, showed normal karyotype. The false positive rate in this population was $2.2 \%$ (3/135). These cases with false positive results of NIPT had no other complications except for the preterm delivery at 32 weeks and 1 day of gestation in one case. Neonatal physical examination was normal in all three cases.
Table 1. Demographic characteristics and abnormal ultrasound findings according to the first-tier choice of genetic test

\begin{tabular}{lccc}
\hline \multicolumn{1}{c}{ Characteristic } & NIPT & Diagnostic genetic & P-value \\
& $(\mathrm{n}=139)$ & test $(\mathrm{n}=206)$ & \\
\hline Age (yr) & $35.7 \pm 3.7$ & $34.4 \pm 4.0$ & 0.44 \\
Singleton pregnancy & $131(94.2)$ & $190(92.2)$ & 0.35 \\
Twin pregnancy & $8(5.8)$ & $16(7.8)$ & 0.01 \\
Gravida & $2.1 \pm 1.2$ & $2.2 \pm 1.4$ & 0.04 \\
Gestational age at & $13.6 \pm 2.9$ & - & \\
$\quad$ sampling (week) & & & \\
Abnormal ultrasound & & & \\
$\quad$ findings & & $92(44.7)$ & 0.25 \\
Increased nuchal & $54(38.8)$ & & \\
$\quad$ translucency & & $44(21.4)$ & 0.96 \\
Structural anomalies & $26(18.7)$ & $70(34.0)$ & 0.22 \\
\hline Soft markers & $59(42.4)$ &
\end{tabular}

Values are presented as mean \pm standard deviation or number (\%).

NIPT, non-invasive prenatal testing; -, not available.

Within the study population, $25(3.0 \%)$ patients had fetal chromosomal abnormalities. The chromosomal aberrations include 20 (80.0\%) cases of common aneuploidy and 5 (20.0\%) cases of other rare chromosomal abnormalities. These 5 (20.0\%) cases of chromosomal aberrations other than common aneuploidies which is not detectable with NIPT were regarded as a residual risk. Overall, when assuming that NIPT was solely performed in the patients with abnormal ultrasound findings, this study showed a $2.2 \%$ of false positive risk and a $20.0 \%$ of residual risk. Fig. 1 shows the flowchart of pregnancies with abnormal ultrasound and Table 2 describes the details of cases with abnormal NIPT results.

This study also analyzed the results according to the type of abnormal ultrasound findings. Abnormal ultrasound findings included INT, structural anomalies, and soft markers. INT was in 146 (42.3\%) pregnancies and 54 (37.0\%) cases chose NIPT as a screening test as shown in the flowchart (Fig. 2). Fifty cases (92.6\%) had low risk results and 4 (7.4\%) cases had high risk results. Of those with high risk results, only 2 cases were confirmed with the diagnostic genetic test. Other 2 cases showed normal karyotype. Ninety-two (45.1\%) cases went through the diagnostic test directly and 14 (15.2\%) cases had chromosomal abnormalities including one case of microdeletion. In pregnancies complicated with INT, false positive rate and residual risk were $3.8 \%$ and $6.3 \%$, respectively.

Isolated or multiple structural anomaly was found in 183 (22.2\%) cases during the study period. For the first-tire test, 26 (14.2\%) cases selected NIPT and 47 (25.7\%) cases selected invasive diagnostic test. Total chromosomal aberration was di- 


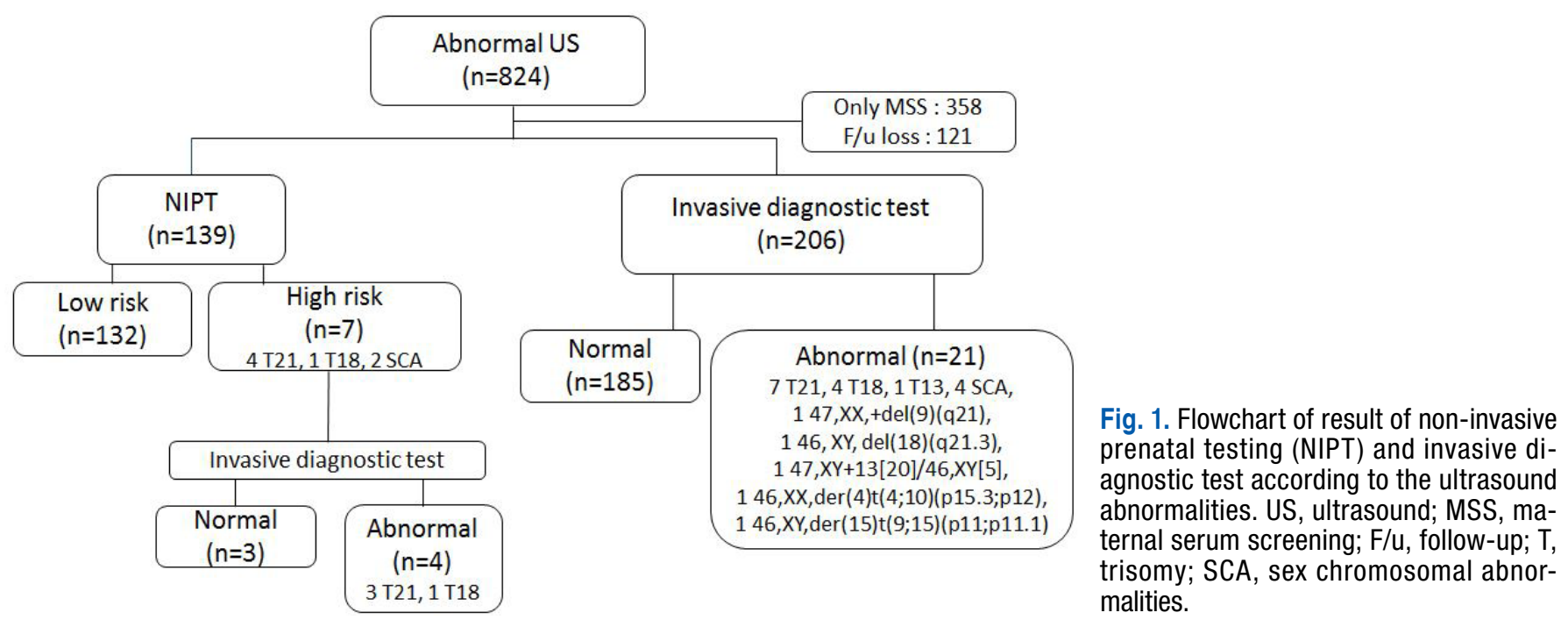

Table 2. Details of cases with abnormal non-invasive prenatal testing (NIPT) results

\begin{tabular}{|c|c|c|c|c|c|c|}
\hline \multirow[b]{2}{*}{ Case } & \multirow[b]{2}{*}{ Indication for genetic testing } & \multicolumn{2}{|c|}{ NIPT } & \multicolumn{2}{|c|}{ Invasive diagnostic testing } & \multirow[b]{2}{*}{ Outcome } \\
\hline & & $\begin{array}{l}\text { GA at sampling } \\
\text { (week) }\end{array}$ & Result & $\begin{array}{l}\text { GA at sampling } \\
\text { (week) }\end{array}$ & Result & \\
\hline 1 & NT $2.92 \mathrm{~mm}$ & $12+0$ & $\mathrm{~T} 18$ & $16+0$ & $47, X Y+18$ & Follow-up loss \\
\hline 2 & NT $2.51 \mathrm{~mm}$ & $11+5$ & T21 & $13+1$ & $47, X X+21$ & Follow-up loss \\
\hline 3 & Hydrops fetalis, short femur, echogenic bowel & $14+0$ & T21 & $16+3$ & $47, X X+21$ & Follow-up loss \\
\hline 4 & Absent nasal bone & $11+3$ & $\mathrm{~T} 21$ & $13+3$ & $47, X Y+21$ & Follow-up loss \\
\hline 5 & NT 2.80 mm & $12+2$ & $45, X$ & $15+2$ & $46, X X$ & PTB 32+1 \\
\hline 6 & $\begin{array}{l}\text { NT } 2.91 \mathrm{~mm} \\
\text { Twin }\end{array}$ & $21+0$ & $\mathrm{~T} 21$ & $24+6$ & $46, X X$ & $\begin{array}{l}\text { 36+1 LB without } \\
\text { congenital anomaly }\end{array}$ \\
\hline 7 & $\begin{array}{l}\text { Prominent nuchal fold, echogenic intracardiac } \\
\text { foci, SGA }\end{array}$ & $16+4$ & $45, x$ & $18+2$ & $46, X X$ & $\begin{array}{l}37+2 \text { LB without } \\
\text { congenital anomaly }\end{array}$ \\
\hline
\end{tabular}

GA, gestational age; NT, nuchal translucency; T, trisomy; PTB, preterm birth; LB, live birth; SGA, small for gestational age.

agnosed in 9 (4.9\%) cases and subchromosomal aberration with translocation and microdeletion was detected in 3 (1.6\%) cases. NIPT showed high risks in 2 cases but the diagnostic result only confirmed one case of T21. As a result, when structural anomalies are present, false positive rate was 4\% and the residual risk was 33.3\%.

However, when ultrasound scan found soft markers, we found only two (1.6\%) chromosomal abnormalities. Among these cases, one case showed mosaicism and this case had two soft markers, which was choroid plexus cyst and echogenic bowel. No other chromosomal abnormalities except for the one T21 case that was also detected with NIPT were found in this study. As a result, when only isolated single soft marker exists, this study population had no false positive or residual risk.

Fig. 2 describes overall NIPT and invasive diagnostic test results according to the ultrasound findings, and Table 3 summarizes the false positive and residual risks of NIPT by type of abnormal ultrasound in this study population.

\section{Discussion}

When fetal abnormalities are found in ultrasound, current recommendations from $\mathrm{ACOG}$ and SMFM advise to perform diagnostic testing with chromosomal microarray due to increased risk of chromosomal abnormalities and discordant results of NIPT $[10,20]$. Nevertheless, some women still consider NIPT as an alternative for diagnostic test and the pretest counseling becomes more important. This study focused to evaluate the clinical efficiency of NIPT in such cases.

This study found the false positive and residual risks of NIPT 


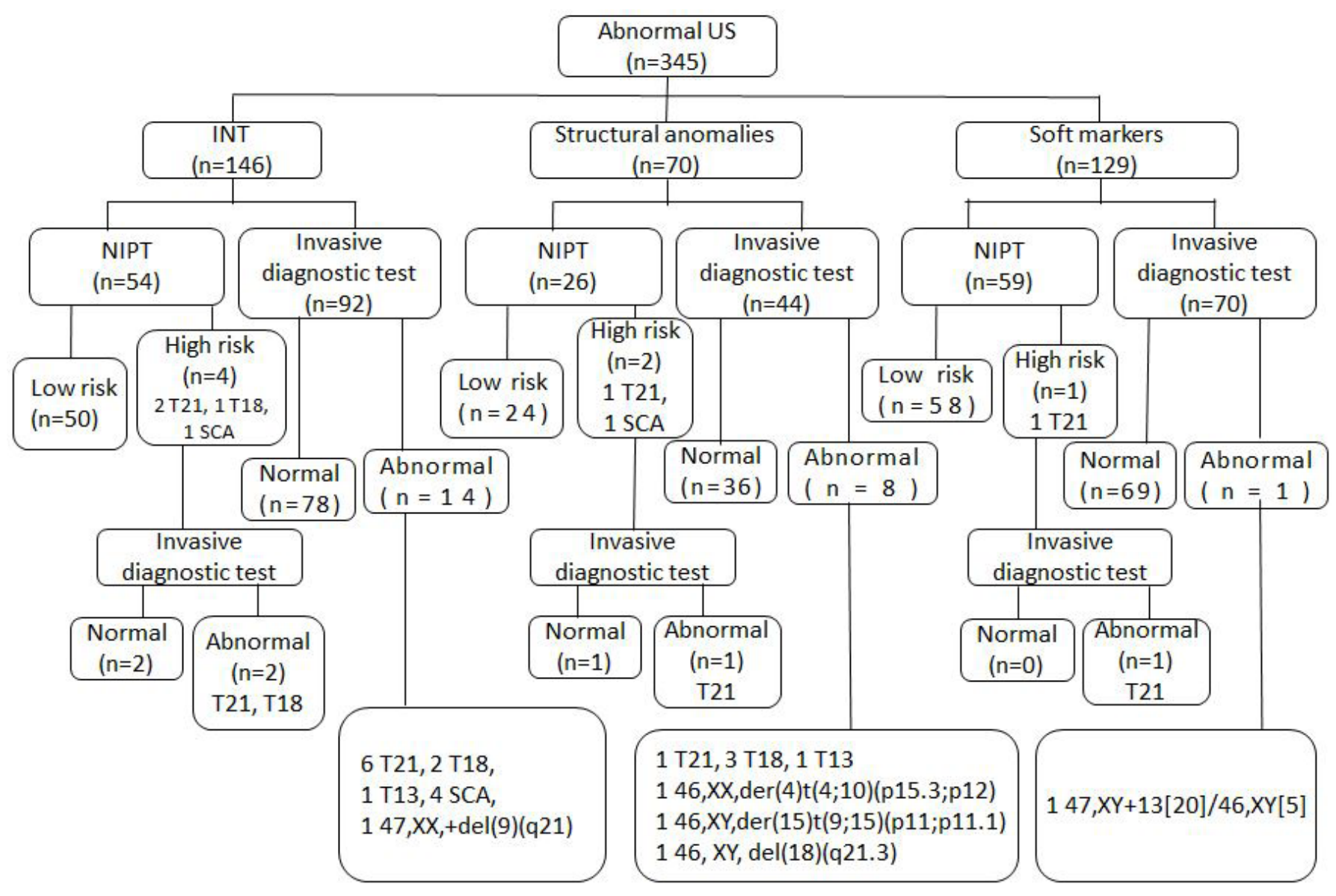

Fig. 2. Flowchart of result of non-invasive prenatal testing (NIPT) and invasive diagnostic test of 345 patients according to the ultrasound abnormalities. US, ultrasound; INT, increased nuchal translucency; T, trisomy; SCA, sex chromosomal abnormalities.

Table 3. Overview of non-invasive prenatal testing (NIPT) results according to the type of abnormality in ultrasound

\begin{tabular}{lcc}
\hline \multirow{2}{*}{ Indication for NIPT } & \multicolumn{2}{c}{ Results } \\
\cline { 2 - 3 } & False positive rate (\%) & Residual risk (\%) \\
\hline Structural anomaly & 4 & 33.3 \\
$\begin{array}{l}\text { Increased nuchal translu- } \\
\text { cency }\end{array}$ & 3.8 & 6.3 \\
Soft marker & \\
$\quad$ Single & & \\
$\geq 2$ markers & 0 & 0 \\
Total & 0 & 50.0 \\
\hline
\end{tabular}

when performed in high risk pregnancies with abnormal ultrasound findings. The discordant results of NIPT signify that patient in this circumstance remains at risk of fetal aneuploidy despite the negative NIPT results. The overall false positive risk and residual risk of NIPT in this study was $2.2 \%$ and $20.0 \%$, respectively. However, the risks differed according to the type of ultrasound findings.

Discordant results of NIPT were most significant when fetal structural anomalies were present. Being concurrent with the previous studies [13,15], the chromosomal abnormalities including the abnormalities that is only detectable through invasive diagnostic test were frequent in this group (4.9\%). Society for Maternal-Fetal Medicine [10] informed that substantial number of fetuses have abnormalities that are not detectable by NIPT when structural abnormalities are present. Likewise, chromosomal abnormalities not detectable with NIPT were frequent in this study group and residual risk was 33.3\%. Other studies also reported additional aneuploidies in patients with abnormal ultrasound findings after negative NIPT results in variable incidence. Benachi et al. [15] reported 7.9\% (23/290) additional pathogenic karyotypes in patient with abnormal ultrasound and normal NIPT results and Beulen et al. [12] reported 3.1\% (7/224) additional clinically relevant genetic aberration. The diagnostic 
test with chromosomal microarray is recognized to detect these additional genetic abnormalities in 6-7\% of normal karyotype fetuses $[10,13,21,22]$. However, 26 patients in this study selected NIPT as a first-tier test after detecting abnormal ultrasound findings, even after the counseling for discordant result and residual risk NIPT. The ultrasound abnormalities included coarctation of the aorta, ventricular septal defect, right atrial isomerism, mitral valve stenosis, aortic stenosis, transposition of great arteries, duodenal atresia, hepatic nodule, abdominal cyst, congenital pulmonary airway malformation, unilateral renal agenesis, polycystic kidney disease, cleft lip and palate, absent nasal bone, club foot, and hydrops fetalis. Most patients in this group chose to try NIPT in fear of procedure-related risk of miscarriage after invasive diagnostic case despite the findings suggesting increased risk fetal aneuploidy, such as duodenal atresia, absent nasal bone, and hydrops fetalis. Two cases had high risk results in NIPT but only one case was confirmed with T21. This case had hydrops fetalis with short femur length. Other cases who underwent NIPT had only isolated structural abnormalities and 8 (30.8\%) cases were confirmed to have no structural abnormality in postnatal evaluation. In this study, most cases with isolated or minor structural abnormalities and false positive prenatal ultrasound results were likely to have chosen NIPT as a first-tier test despite structural abnormalities.

The group with INT also showed meaningful false positive and residual risk in this study. Since INT has been associated with structural anomalies, neuromuscular disorders, and genetic conditions, the prevalence of chromosomal abnormalities increases as in the group with structural abnormalities [10]. These underlying factors also increases the residual risk of chromosomal aberrations in patients with negative NIPT result. Previous study by Khalil et al. [14] reported the residual risk of a significant chromosomal abnormality after negative NIPT results in patient with fetal INT as 2.5\%. The residual risk was 6.3\% in this study population. Therefore, when INT is detected in the first trimester of pregnancy, the counseling for further screening or diagnostic test should contain information about the residual risk and discordant result of NIPT.

However, in cases with isolated single soft marker, there was no false positive or residual risk in this study. This result corresponds to the previous studies stating that isolated soft markers having a limited utility in the detection of the aneuploidies $[10,23]$. These studies also reported low residual risk of NIPT in patients with soft markers and recommended to state isolated soft markers with normal NIPT results as normal variants or findings with no clinical significance. However, there was one case of chromosomal abnormality that was not detectable by NIPT in our study and the fetus of the case had 2 soft markers which was choroid plexus cyst and echogenic bowel. The likelihood of aneuploidy is known to be higher when more than one soft marker is found, but the actual risks again differ according to the specific soft markers found. Because of the complexity with multiple soft markers, the counseling is suggested to include consideration for the diagnostic test $[10,24]$. As a result of our study and previous recommendations, NIPT might be an acceptable testing for the patients with a single isolated soft marker.

In this study, we also explained NIPT to women with twin pregnancy as a testing option. The current recommendations discouraging NIPT in multifetal gestations with fetal anomaly [9] were explained but 8 cases underwent NIPT. Six cases had INT in one or both fetuses and 2 cases had isolated single soft marker. Fortunately, there was no false negative results found but one case with INT showed false positive results and had to go through the invasive diagnostic test. The discordant results in twin pregnancy was not enough in this study population, but the limited efficiency of NIPT in multiple pregnancy should be emphasized when counseling the patients.

Fortunately, there were no definite false negative results in this study population. No phenotype or functional anomalies were found in the neonate and parents in study population with low risk NIPT result. Five cases in this study had NIPT in first trimester and had invasive diagnostic test due to abnormal ultrasound findings in second trimester, despite negative NIPT results. The karyotype was normal in all 5 cases. The risk of false negative is always a concern for the NIPT, and the physicians and counselors should always consider the residual risks and beware of falsely reassuring results. However, not all neonates have gone through the genetic test in this study and the chromosomal microarray was not routinely performed during the diagnostic test in our center during this study period, leaving more possible undetected residual chromosomal abnormalities. These are the limitation of this study. For this reason, the residual risk calculated in this study might not reflect the risk in the whole population. Furthermore, since this study focused on the abnormal ultrasound findings, other baseline high risks such as advanced maternal age and previous history of aneuploidy were not considered. Further studies considering effect of each of the factors could be more helpful.

In conclusion, this study found discordant results between NIPT and invasive diagnostic testing in pregnancy with abnormal ultrasound findings. However, the false positive or the residual risks of NIPT differed according to the type of ultrasound 
abnormalities. The discordancy was significant with structural anomalies but not found in cases with single soft marker. As a result, it could be possible to consider NIPT as alternative to the invasive diagnostic test in certain circumstances. Moreover, as efficacy of the NIPT differs with the ultrasound findings, the counseling for prenatal screening or diagnostic test should be individualized according to the ultrasound results and other underlying individual risks.

\section{Acknowledgements}

The publication of this article was funded by grants ( $\mathrm{HC} 15 \mathrm{C}$ 1336) from the Korea Health Technology R\&D Project through the Korea Health Industry Development Institute (KHIDI), funded by the Ministry of Health \& Welfare, Republic of Korea. No other source of economic support was received.

\section{References}

1. Gil MM, Quezada MS, Revello R, Akolekar R, Nicolaides KH. Analysis of cell-free DNA in maternal blood in screening for fetal aneuploidies: updated meta-analysis. Ultrasound Obstet Gynecol 2015;45:249-66.

2. Norton ME, Jacobsson $B$, Swamy GK, Laurent $L C$, Ranzini $A C, B r a r H_{\text {, }}$ et al. Cell-free DNA analysis for noninvasive examination of trisomy. N Eng J Med 2015;372:1589-97.

3. Vora NL, Robinson S, Hardisty EE, Stamilio DM. Utility of ultrasound examination at 10-14 weeks prior to cell-free DNA screening for fetal aneuploidy. Ultrasound Obstet Gynecol 2017;49:465-9.

4. Reiff ES, Little SE, Dobson L, Wilkins-Haug L, Bromley B. What is the role of the 11- to 14-week ultrasound in women with negative cellfree DNA screening for aneuploidy? Prenat Diagn 2016;36,260-5.

5. Larion S, Warsof SL, Romary L, Mlynarczyk M, Peleg D, Abuhamad AZ. Uptake of noninvasive prenatal testing at a large academic referral center. Am J Obstet Gynecol 2014;211:651.e1-7.

6. Larion S, Warsof SL, Romary L, Mlynarczyk M, Peleg D, Abuhamad AZ. Use of the combined first-trimester screen in high-and low-risk patient populations after introduction of noninvasive prenatal testing. J Ultrasound Med 2015;34:1423-8.

7. Warsof SL, Larion S, Abuhamad AZ. Overview of the impact of noninvasive prenatal testing on diagnostic procedures. Prenat Diagn 2015; 35:972-9.

8. Norton ME, Jelliffe-Pawlowski LL, Currier RJ. Chromosome abnormalities detected by current prenatal screening and noninvasive prenatal testing. Obstet Gynecol 2014;124:979-86.

9. Committee on Practice Bulletins-Obstetrics, Committee on Genetics, and the Society for Maternal-Fetal Medicine. Practice bulletin no.
163: screening for fetal aneuploidy. Obstet Gynecol 2016;127:e12337.

10. Society for Maternal-Fetal Medicine (SMFM), Norton ME, Biggio JR, Kuller JA, Blackwell SC. The role of ultrasound in women who undergo cell-free DNA screening. Am J Obstet Gynecol 2017;216:B2-7.

11. American College of Obstetricians and Gynecologists. Committee opinion no. 640: cell-free DNA screening for fetal aneuploidy. Obstet Gynecol 2015;126:e31-7.

12. Beulen L, Faas BHW, Feenstra I, Van Vugt JMG, Bekker MN. Clinical utility of non-invasive testing in pregnancies with ultrasound anomalies. Ultrasound Obstet Gynecol 2017;49:721-8.

13. Wapner RJ, Martin CL, Levy B, Ballif BC, Eng CM, Zachary JM, et al. Chromosomal microarray versus karyotyping for prenatal diagnosis. N Engl J Med 2012;367:2175-84.

14. Khalil A, Mahmoodian N, Kulkarni A, Homfray T, Papageorghiou A, Bhide $A$, et al. Estimation of detection rates of aneuploidy in highrisk pregnancy using an approach based on nuchal translucency and non-invasive prenatal testing: a cohort study. Fetal Diagn Ther 2015; 38:254-61.

15. Benachi $A$, Letourneau $A$, Kleinfinger $P$, Senat MV, Gautier E, Favre $R$, et al.; Collaborative SEquençage a Haut Debit et Aneuploidies (SEHDA) Study Group. Cell-free DNA analysis in maternal plasma in cases of fetal abnormalities detected on ultrasound examination. Obstet Gynecol 2015;125:1330-7.

16. Salomon $\sqcup$, Alfirevic Z, Bilardo CM, Chalouhi GE, Ghi T, Kagan KO, et al. ISUOG practice guidelines: performance of first-trimester fetal ultrasound scan. Ultrasound Obstet Gynecol 2013;41:102-13.

17. Chung JH, Yang JH, Song MJ, Cho JY, Lee YH, Park SY, et al. The distribution of fetal nuchal translucency thickness in normal Korean fetuses. J Korean Med Sci 2004;19:32-6.

18. Norton ME, Rink BD. Genetics and prenatal genetic testing. In: Callen PW, Norton ME, Scoutt LM, Feldstein VA, eds. Callen's ultrasonography in obstetrics and gynecology. 6th ed. Philadelphia: Elsevier, 2017; 43.

19. Cunningham FG, Leveno KJ, Bloom SL, Dashe JS, Hoffman BL, Casey BM, et al. Williams obstetrics. 25th ed. New York: McGraw-Hill Education, 2018. p. 182-224.

20. Committee on Genetics and the Society for Maternal-Fetal Medicine. Committee opinion no.682: microarrays and next-generation sequencing technology: the use of advanced genetic diagnostic tools in obstetrics and gynecology. Obstet Gynecol 2016;128:e262-8.

21. Shaffer LG, Rosenfeld JA, Dabell MP, Coppinger J, Bandholz AM, Ellison JW, et al. Detection rates of clinically significant genomic alterations by microarray analysis for specific anomalies detected by ultrasound. Prenat Diagn 2012;32:986-95.

22. Hillman SC, McMullan DJ, Hall G, Togneri FS, James N, Maher EJ, et al. 
Use of prenatal chromosomal microarray: prospective cohort study and systematic review and meta-analysis. Ultrasound Obstet Gynecol 2013;41:610-20.

23. Agathokleous $M$, Chaveeva $P$, Poon LC, Kosinski $P$, Nicolaides KH. Meta-analysis of second-trimester markers for trisomy 21. Ultrasound
Obstet Gynecol 2013;41:247-61.

24. Committee on Practice Bulletins-Obstetrics and the American Institute of Ultrasound in Medicine. Practice bulletin no. 175: ultrasound in pregnancy. Obstet Gynecol 2016;128:e241-56. 